\title{
Influence of Plant Growth Regulators on Micropropagation of Gwarpatha [Aloe vera (L.) Burm.]
}

\author{
Mohan Lal Jakhar*, Divya Dixit, Sarfraz Ahmad, Mahaveer Prasad Ola, \\ Manohar Ram and Hari Ram Jat
}

\begin{abstract}
Department of Plant Breeding and Genetics, S.K.N. Agriculture University, Jobner, Rajasthan-303029, India
\end{abstract}

*Corresponding author

Keywords

Aloe vera, Gwarpatha, Micropropagation, Plant growth regulator, Root induction, Shoot proliferation, Hardening.

\section{Article Info}

Accepted:

12 February 2020

Available Online: xx March 2020

\section{A B S T R A C T}

Present investigation was carried out to determine the optimum level of different plant growth regulators for rapid shoot multiplication, rooting and hardening of micropropagated plantlets of Aloe vera. The experiment was laid out using completely randomized design (CRD) with ten replications during 2018-19. Auxiliary shoot explants were inoculated on MS medium supplemented with various concentrations of cytokinin (BAP $0.5-6.0 \mathrm{mg} / \mathrm{l}$ ) alone and BAP (0.5-6.0 $\mathrm{mg} / \mathrm{l}$ ) with auxin (NAA $0.1-0.6$ ) in combination for direct shoot bud proliferation. For rooting, auxins (NAA/IBA) were used at the concentrations of $0.5-2.0 \mathrm{mg} / \mathrm{l}$ singly and $0.5-2.0 \mathrm{mg} / \mathrm{l}$ NAA with $\mathrm{IBA}$ at 0.5 and $2.0 \mathrm{mg} / \mathrm{l}$ in combinations. Maximum shoot bud induction was obtained when MS medium supplemented with $4.0 \mathrm{mg} / \mathrm{l} \mathrm{BAP}$ singly and $4.5 \mathrm{mg} / \mathrm{l} \mathrm{BAP}$ with $0.6 \mathrm{mg} / \mathrm{l} \mathrm{NAA}$ in combination. Maximum rooting was induced at $1.5 \mathrm{mg} / \mathrm{IBA}$ and $0.5 \mathrm{mg} / \mathrm{l} \mathrm{IBA}$ with $2.0 \mathrm{mg} / \mathrm{l} \mathrm{NAA}$ in combination. The rooted plantlets showed 80 per cent survival in culture room and 74 per cent in shade house during the process of hardening. Further, it is recommended that the given protocols may be used for mass multiplication of Aloe vera to meet the demand of farmers as well as pharmaceutical industries.

\section{Introduction}

Desert medicinal plant Gwarpatha (Aloe vera L. syn. Aloe barbadensis Miller) belongs to family Asphadelaceae (Souza and Lorenzi 2005). It is an ancient, semi tropical medicinal plant indigenous to Africa, Madagascar and Arabia and introduced plant in India. This genus has more than four hundred species of flowering succulent plants. Aloe vera is coarse looking evergreen perennial plant, grows up to $80-100 \mathrm{~cm}$ in height, with a strong fibrous root and at large stem supporting a rosette of narrow lanceolate leaves. The leaves are whitish green on both sides and bear spiny teeth on the margins. The 
yellow to orange drooping flowers produced in an inflorescence of 90-110 cm tall, each flower is pendulous, with yellow tubular corolla 2-3 cm and hermaphrodite. It has valuable medicinal properties and is commercially used in pharmaceuticals, cosmetics, food industries, as nutraceuticals and for many herbal preparations. There are about more than 40 Aloe based formulations being marketed in the global market. In India, tissue culture research began nearly six decades ago with the first report on production of test tube fertilization. Plant cell and tissue culture is defined as the capability to regenerate and propagate plants from single cells, tissues and organs under sterile and controlled environmental conditions (Murashige and Skoog, 1962). Tissue culture methods have also been employed to study the basic aspects of plant growth, metabolism, differentiation and morphogenesis and provide ideal opportunity to manipulate these processes. Advancement in tissue cultural methodology led many recalcitrant plants amenable to in vitro regeneration and to the development of haploids, somatic hybrids and pathogen free plants (Gupta et al., 2014).

In nature (in vivo), Aloe vera is propagated through lateral buds which are slow, very expensive and low income practice (Meyer and Staden, 1991). Sexual reproduction by seeds due to male sterility in Aloe vera is almost ineffective and vegetative propagation through lateral shoots only possible during growing seasons (Keijzer and Cresti, 1987). Thus large scale plantation of Aloe vera through in vivo system of plantlet multiplication is insufficient to meet the requirements of farmers and pharmaceutical industries demand (Bhandari et al., 2010). There is a need to develop suitable and alternative method for large scale propagation for rapid plant multiplication to meet the demand of farmers and pharmaceutical industries (Abrie and Staden, 2001).
Therefore, the present study aimed to develop an alternative protocol for rapid and high frequency in vitro propagation of Aloe vera, looking through its increasing demand for pharmaceutical industry at global level.

\section{Materials and Methods}

The present investigation was carried out at Tissue Culture Laboratory of Department of Plant Breeding and Genetics, S.K.N. Agriculture University, Jobner, Rajasthan, India during 2018-19.

\section{Plant growth regulators}

Different concentrations of plant growth regulators were incorporated singly and in combinations in the MS medium for direct shoot proliferation from auxiliary explants.

1. Plant growth regulators incorporated singly in the medium: BAP: 0.5 , 1.0, 1.5, 2.0, 2.5, 3.0, 3.5, 4.0, 4.5, $5.0,5.5$ and $6.0 \mathrm{mg} / 1$

2. Plant growth regulators incorporated in combinations: BAP: $(0.5,1.0$, 1.5, 2.0, 2.5, 3.0, 3.5, 4.0, 4.5, 5.0, 5.5 and $6.0 \mathrm{mg} / \mathrm{l})+\mathrm{NAA}:(0.1$, $0.2,0.3,0.4,0.5$ and $0.6 \mathrm{mg} / \mathrm{l})$ in all possible combinations.

\section{Induction of roots}

Explants were inoculated for induction of roots in the medium supplemented with different concentrations of plant growth regulators added alone or in combinations.

1. Plant growth regulators incorporated singly in the medium NAA/IBA: 0.5, 1.0, 1.5 and $2.0 \mathrm{mg} / \mathrm{l}$.

2. Plant growth regulators incorporated in combinations:

NAA: $(0.5,1.0,1.5$ and $2.0 \mathrm{mg} / \mathrm{l})+$ IBA: (0.5 and $2.0 \mathrm{mg} / \mathrm{l})$

MS basal medium without supplementation of any growth regulator was used as a control in 
all the experiments. All the cultures were maintained in an air conditioned culture room at a temperature of $25 \pm 2{ }^{\circ} \mathrm{C}$ under fluorescent light in a 14:10 hours' photoperiod.

\section{Acclimatization of plantlets}

After 40-50 days of culture on rooting media, the plantlets were shifted to polythene bags/earthen pots for their hardening prior to final transfer to soil in natural conditions. After removing media, these were dipped in $1 \% \mathrm{w} / \mathrm{v}$ solution of Bavistin to prevent any fungal infection to newly developed plants. After Bavistin treatment the plantlets were carefully planted in polythene bags/earthen pots containing 1:1 mixture of soil and vermicompost. Then the plants were shifted to shade house with indirect sunlight. After 5060 days, shade house plants were finally transferred to the field.

\section{Observations recorded}

Observations on period of initiation of shoot, number of shoots per explant, morphogenetic response (Percent), shoots length $(\mathrm{cm})$, number of days taken for root induction, number of roots, root length $(\mathrm{cm})$, mean days taken for callus initiation, colour and texture of the callus are recorded following standard procedures.

\section{Statistical analysis}

Each experiment was conducted in completely randomized design and data were analyzed for means and standard error accordingly as described by Snedecor and Cochran (1972). Standard error was calculated only after value transformation for the characters where response was less than 100 per cent. The value for each replication was transformed by square root transformation as follows

$$
\sqrt{Y+1 / 2}
$$

Where, $\mathrm{Y}=$ original value

Tests of significance were done according to Duncan's Multiple Range Test (DMRT) for different traits (Gomez and Gomez, 1984).

\section{Results and Discussion}

\section{Effect of plant growth regulators on shoot proliferation}

BAP was used as cytokinin for shoot bud proliferation in the present investigation. The medium devoid of growth regulators failed to initiate bud break or callus formation from any of the explant. BAP when incorporated singly in the basal MS medium, induced multiple shoot buds at all the levels (Table 1), however the most profuse shoot bud breaks (12.8) and shoot length $(8.9 \mathrm{~cm})$ were obtained at $4.0 \mathrm{mg} / \mathrm{l} \mathrm{BAP}$ (Fig. 1) followed by $6.0 \mathrm{mg} / \mathrm{l}$ BAP (11.2). Slight to medium loose/semi compact callus was also induced at the base of auxiliary explants incubated at different levels of BAP (1.5-2.5 mg/l). Lower (0.5-1.0 mg /1) and higher (3.0-6.0) levels of BAP inhibited callus differentiation. These results were in close agreement with other reports of micropropagation of Aloe vera (Ahmed et al., 2007 and Dwivedi et al., 2014).

Eufrocinio and Malasa (2005) in $A$. barbadensis reported best multiple shoot bud induction at medium containing $1.0 \mathrm{mg} / \mathrm{l}$ BAP. However, these results were contrary to the results of present investigation with the level of BAP might be due to differences in species and explants used in the particular study. Callusing was also observed at the base of auxiliary explant in present investigation which was similar to the observations of Kawai et al., 2006 and Yadav, 2008 in Aloe arborescens and Aloe vera, respectively. Auxin (BAP) combined with cytokinin 
(NAA) enhanced the shoot bud multiplication considerably (Table 2). Highest shoot proliferation (12.6) was observed at medium supplemented BAP $(4.5 \mathrm{mg} / \mathrm{l})$ with NAA (0.6 $\mathrm{mg} / \mathrm{l}$ ) (Fig. 2). These were at par with the level of BAP $(4.0 \mathrm{mg} / \mathrm{l})$ where maximum shoot proliferation was observed upon supplementation in the medium alone. Callus formation was completely inhibited at the base of explants in the medium supplemented with combination of BAP and NAA.

These results are in accordance with the results obtained by Khanam et al., (2014). They reported that a perfect combination of auxin and cytokinin is needed for optimum shoot induction. MS basal medium in combination with $4.0 \mathrm{mg} / \mathrm{l} \mathrm{BAP}$ and $0.2 \mathrm{mg} / \mathrm{l}$ NAA was found to be the best on which explants began to show emergence of shoot buds within one week. Similar observations were also found by Nayankantha et al., (2010) and Kiran et al., (2017), who reported synergetic effect of BAP and NAA in shoot bud break.

\section{Effect of plant growth regulators on root induction}

Root induction was observed at all the levels of IBA and NAA added either singly or in combination with 100 per cent frequency (Table 3). Maximum number of root induction (6.6) was obtained in the medium supplemented with $1.5 \mathrm{mg} / \mathrm{l} \mathrm{IBA}$ with shoot length $(7.64 \mathrm{~cm})$ followed by $(5.4) 1.0 \mathrm{mg} / \mathrm{l}$ IBA (Fig. 3). In IBA supplemented media, roots were thin and long, whereas thick roots were induced in medium supplemented with NAA (Fig. 4).

In combination, maximum number of roots (5.6) was observed at $0.5 \mathrm{mg} / \mathrm{l} \mathrm{IBA}$ with 2.0 $\mathrm{mg} / 1 \mathrm{NAA}$ with longest root length $(8.3 \mathrm{~cm})$ followed by $0.5 \mathrm{mg} / \mathrm{lBA}+1.5 \mathrm{mg} / \mathrm{l} \mathrm{NAA}$ (Fig. 5). In comparison to NAA, IBA produced higher number of roots at all the levels except at $2.0 \mathrm{mg} / \mathrm{l} \mathrm{IBA}$. There was significant difference among the levels of IBA and NAA for number of root induction per explant. Results of present investigation were similar to the reports of Abrie and Staden (2001) in Aloe polyphylla with respect to type of plant growth regulator (IBA), they observed maximum root on medium supplemented with $0.5 \mathrm{mg} / \mathrm{l} \mathrm{IBA}$.

However, in the present study maximum root induction was ovserved at $1.5 \mathrm{mg} / \mathrm{l} \mathrm{IBA}$. This might be due to difference in the species under study. Hashemabadi and Kaviani (2008) found the best root induction at MS medium supplemented with $1.0 \mathrm{mg} / \mathrm{l} \mathrm{IBA}$ with $1.0 \mathrm{mg} / \mathrm{l}$ NAA. Khanam et al., (2014) reported profuse root induction after one week of culture at $2.0 \mathrm{mg} / \mathrm{l} \mathrm{IBA}$ and $1.0 \mathrm{mg} / \mathrm{l}$ NAA. However, these levels were not tested in the present investigation.

\section{Acclimatization of plantlets}

After 40-50 days of culture of in vitro developed shoots on most responsive rooting media were shifted to pots filled with mixture of 1:1 ratio of soil and vermicompost for their hardening prior to final transfer to soil, showed good percentage of survival (80 per cent) in culture room (Fig. 6). In shade house plants also showed 74 per cent survival rate (Fig. 7).

The growth and elongation of plants were less in culture room whereas, in shade house growth of plants was better and they also started to elongation and thickening of leaves in shade house. Perusal of literature on micropropagation of Aloe also indicated that rooting and hardening had never been encountered as a problem in Aloe's tissue culture. Various researchers have reported successfully in vitro rooting and hardening of Aloe vera (Kalimuthu et al., 2010 and Rathore et al., 2011) and corroborate present study. 
Table.1 Morphogenetic effect of various concentrations of Cytokinin (BAP) added singly in the MS medium on auxiliary explant of Aloe vera.

\begin{tabular}{|c|c|c|c|c|c|c|c|c|c|}
\hline \multirow[t]{2}{*}{ S.N. } & \multirow[t]{2}{*}{$\begin{array}{c}\text { BAP } \\
(\mathrm{mg} / \mathrm{l})\end{array}$} & \multirow{2}{*}{$\begin{array}{c}\text { Days taken } \\
\text { in shoot } \\
\text { initiation }\end{array}$} & \multirow{2}{*}{$\begin{array}{c}\text { Morphogeneti } \\
\text { c response } \\
(\%)\end{array}$} & \multicolumn{2}{|c|}{$\begin{array}{c}\text { Number of shoot buds induction per } \\
\text { explant }\end{array}$} & \multirow[t]{2}{*}{$\begin{array}{l}\text { Shoot length } \\
(\mathrm{cm})\end{array}$} & \multirow{2}{*}{$\begin{array}{c}\text { Days to } \\
\text { callus } \\
\text { initiation }\end{array}$} & \multirow[t]{2}{*}{$\begin{array}{l}\text { Colour of } \\
\text { callus }\end{array}$} & \multirow[t]{2}{*}{$\begin{array}{l}\text { Texture } \\
\text { of callus }\end{array}$} \\
\hline & & & & $\begin{array}{l}6 \text { weeks after } \\
\text { inoculation }\end{array}$ & $\begin{array}{l}8 \text { weeks after } \\
\text { inoculation }\end{array}$ & & & & \\
\hline 1. & 0.5 & 18.1 & 100 & $2.1 \pm 0.18 \quad \mathbf{f}$ & $3.8 \pm 0.25 \quad \mathbf{g}$ & $3.32 \pm 0.36 \mathbf{e}$ & - & - & - \\
\hline 2. & 1.0 & 16.1 & 100 & $2.2 \pm 0.25 \quad \mathbf{f}$ & $4.1 \pm 0.28 \quad$ fg & $4.55 \pm 0.38 \quad$ d & - & - & - \\
\hline 3. & 1.5 & 14.6 & 100 & $2.6 \pm 0.16 \quad \mathbf{f}$ & $4.4 \pm 0.16 \quad$ fg & $5.33 \pm 0.36 \quad d$ & $22.1[40]$ & $\begin{array}{l}\text { Yellowish } \\
\text { green }\end{array}$ & Loose \\
\hline 4. & 2.0 & 15.3 & 100 & $3.4 \pm 0.16$ e & $5.2 \pm 0.33$ ef & $6.68 \pm 0.40 \quad$ c & $24.6[20]$ & $\begin{array}{l}\text { Yellowish } \\
\text { green }\end{array}$ & Loose \\
\hline 5. & 2.5 & 15.9 & 100 & $3.6 \pm 0.27 \quad \mathbf{e}$ & $6.1 \pm 0.28 \quad$ de & $8.3 \pm 0.40 \quad$ b & $28.5[10]$ & $\begin{array}{l}\text { Light } \\
\text { green }\end{array}$ & $\begin{array}{c}\text { Semi } \\
\text { compact }\end{array}$ \\
\hline 6. & 3.0 & 15.3 & 100 & $3.8 \pm 0.25$ e & $6.8 \pm 0.25 \quad$ d & $8.55 \pm 0.38 \quad$ b & - & - & - \\
\hline 7. & 3.5 & 14.2 & 100 & $5.2 \pm 0.25 \quad$ d & $8.4 \pm 0.45 \quad$ c & $8.9 \pm 0.42 \quad$ b & - & - & - \\
\hline 8. & 4.0 & 12.5 & 100 & $8.4 \pm 0.16$ a & $12.8 \pm 0.39 \mathbf{a}$ & $11.16 \pm 0.37 \mathbf{a}$ & - & - & - \\
\hline 9. & 4.5 & 14.4 & 100 & $6.2 \pm 0.25$ c & $9.2 \pm 0.44 \quad$ c & $4.92 \pm 0.37 \quad$ d & - & - & - \\
\hline 10. & 5.0 & 11.5 & 100 & $6.3 \pm 0.26$ c & $8.4 \pm 0.45 \quad$ c & $2.05 \pm 0.27 \quad \mathbf{f}$ & - & - & - \\
\hline 11. & 5.5 & 12.2 & 100 & $7.1 \pm 0.18 \quad$ b & $10.4 \pm 0.34 \mathbf{b}$ & $1.62 \pm 0.14 \mathbf{f}$ & - & - & - \\
\hline 12. & 6.0 & 13.2 & 100 & $6.2 \pm 0.25$ c & $11.2 \pm 0.77 \mathbf{b}$ & $1.47 \pm 0.14$ f & - & - & - \\
\hline
\end{tabular}

Values followed by same letters in each column are not significantly different $(\mathrm{p}<0.05)$ using DMRT

[ ] = Value in parenthesis represents percentage of response $(-)=$ No Response 
Table.2 Morphogenetic effect of various concentrations of Cytokinin (BAP) and Auxin (NAA) added in combinations in the MS medium on auxiliary explant of Aloe vera

\begin{tabular}{|c|c|c|c|c|c|c|}
\hline \multirow{2}{*}{$\begin{array}{l}\text { S. } \\
\text { N. }\end{array}$} & \multirow{2}{*}{$\begin{array}{c}(\mathbf{B A P}+\mathrm{NAA}) \\
(\mathrm{mg} / \mathrm{l})\end{array}$} & \multirow{2}{*}{$\begin{array}{c}\text { Days } \\
\text { taken in } \\
\text { shoot } \\
\text { initiation }\end{array}$} & \multirow{2}{*}{$\begin{array}{l}\text { Morphogenetic } \\
\text { response } \\
(\%)\end{array}$} & \multicolumn{2}{|c|}{ Number of shoot buds per explant } & \multirow{2}{*}{$\begin{array}{l}\text { Shoot length } \\
(\mathrm{cm})\end{array}$} \\
\hline & & & & $\begin{array}{l}6 \text { weeks after } \\
\text { inoculation }\end{array}$ & $\begin{array}{l}8 \text { weeks after } \\
\text { inoculation }\end{array}$ & \\
\hline 1. & $0.5+0.1$ & 18.2 & 100 & $2.2 \pm 0.25 \mathbf{x}$ & $3.8 \pm 0.249$ ab & $3.15 \pm 0.33$ qrstuv \\
\hline 2. & $1.0+0.1$ & 17.1 & 100 & $2.3 \pm 0.21 \quad \mathbf{w x}$ & $4.2 \pm 0.249$ aaab & $3.31 \pm 0.33$ pqrstu \\
\hline 3. & $1.5+0.1$ & 16.2 & 100 & $2.6 \pm 0.16 \mathbf{v w x}$ & $4.4 \pm 0.163 \quad$ zaaab & $4.09 \pm 0.39$ mnopqr \\
\hline 4. & $2.0+0.1$ & 15.3 & 100 & $4.2 \pm 0.25$ pqrs & $5.2 \pm 0.2 \quad$ xyzaa & $5.24 \pm 0.43 \quad$ jklm \\
\hline 5. & $2.5+0.1$ & 15.8 & 100 & $4.3 \pm 0.21$ opqrs & $6.4 \pm 0.34 \quad$ stuvw & $6.99 \pm 0.22$ efgh \\
\hline 6. & $3.0+0.1$ & 15.2 & 100 & $4.4 \pm 0.34$ nopqr & $6.6 \pm 0.221$ rstuv & $7.5 \pm 0.41 \quad$ def \\
\hline 7. & $3.5+0.1$ & 13.8 & 100 & $4.6 \pm 0.27$ mnopq & $7.6 \pm 0.34 \quad$ opqr & $7.95 \pm 0.43$ cdef \\
\hline 8. & $4.0+0.1$ & 12.4 & 100 & $5.4 \pm 0.27 \mathbf{~ k l m}$ & $8.8 \pm 0.327 \quad \mathbf{j k} \mathbf{l m}$ & $9.16 \pm 0.43 \mathbf{a}$ \\
\hline 9. & $4.5+0.1$ & 12.1 & 100 & $6.4 \pm 0.22$ hij & $9.6 \pm 0.427$ ghijk & $4.93 \pm 0.37 \mathbf{j k k l m n}$ \\
\hline 10. & $5.0+0.1$ & 11.3 & 100 & $7.2 \pm 0.2$ efgh & $10.2 \pm 0.416$ fghi & $1.95 \pm 0.23 \mathbf{v w x}$ \\
\hline 11. & $5.5+0.1$ & 12.3 & 100 & $7.2 \pm 0.29$ efgh & $9.2 \pm 0.359 \quad$ ijkklm & $1.67 \pm 0.18 \mathbf{w x}$ \\
\hline 12. & $6.0+0.1$ & 13.2 & 100 & $8.4 \pm 0.31$ cd & $10.4 \pm 0.221$ fgh & $1.58 \pm 0.14 \mathrm{x}$ \\
\hline 13. & $0.5+0.2$ & 18.4 & 100 & $3.2 \pm 0.25$ tuvw & $5.2 \pm 0.249$ xyzaa & $2.69 \pm 0.26$ stuvwx \\
\hline 14. & $1.0+0.2$ & 16.9 & 100 & $2.4 \pm 0.16 \mathbf{w x}$ & $5.6 \pm 0.267 \mathbf{v w x y}$ & $3.48 \pm 0.29$ opqrst \\
\hline 15. & $1.5+0.2$ & 15.9 & 100 & $3.2 \pm 0.25$ tuvw & $6.2 \pm 0.327$ tuvwx & $4.26 \pm 0.39$ mnopq \\
\hline 16. & $2.0+0.2$ & 15.1 & 100 & $3.2 \pm 0.25$ tuvw & $7.2 \pm 0.2$ qrst & $5.74 \pm 0.38$ ijkkl \\
\hline 17. & $2.5+0.2$ & 14.6 & 100 & $3.6 \pm 0.27$ rstu & $7.4 \pm 0.221$ pqrs & $7.0 \pm 0.26$ efgh \\
\hline 18. & $3.0+0.2$ & 13.9 & 100 & $4.2 \pm 0.2$ pqrs & $7.8 \pm 0.327$ nopq & $7.84 \pm 0.42$ cdef \\
\hline 19. & $3.5+0.2$ & 13.3 & 100 & $5.2 \pm 0.2$ Imno & $7.2 \pm 0.359$ qrst & $7.58 \pm 0.36$ def \\
\hline 20. & $4.0+0.2$ & 12.9 & 100 & $6.2 \pm 0.25 \mathbf{i j k}$ & $8.6 \pm 0.34$ klmn & $9.83 \pm 0.45 \mathbf{a b}$ \\
\hline 21. & $4.5+0.2$ & 11.9 & 100 & $6.6 \pm 0.36$ ghij & $9.2 \pm 0.389$ ijkklm & $5.72 \pm 0.38 \mathbf{~ i j k l}$ \\
\hline 22. & $5.0+0.2$ & 11.1 & 100 & $7.2 \pm 0.2$ efgh & $9.4 \pm 0.306$ hijkk & $2.39 \pm 0.24$ yuvwx \\
\hline 23. & $5.5+0.2$ & 13.3 & 100 & $8.4 \pm 0.4 \mathbf{c d}$ & $10.2 \pm 0.249$ fghi & $1.92 \pm 0.24 \mathbf{v w x}$ \\
\hline 24. & $6.0+0.2$ & 13.1 & 100 & $9.8 \pm 0.44 \mathbf{a}$ & $12.2 \pm 0.291 \mathbf{e}$ & $1.57 \pm 0.16 \mathbf{x}$ \\
\hline
\end{tabular}




\begin{tabular}{|c|c|c|c|c|c|c|}
\hline 25. & $0.5+0.3$ & 18.6 & 100 & $2.4 \pm 0.16 \mathbf{w x}$ & $5.4 \pm 0.163 \mathbf{w x y z}$ & $2.97 \pm 0.36$ qrstuvw \\
\hline 26. & $1.0+0.3$ & 17.4 & 100 & $2.6 \pm 0.16 \mathbf{v w x}$ & $5.8 \pm 0.249$ uvwxy & $2.85 \pm 0.30$ rstuvw \\
\hline 27. & $1.5+0.3$ & 16.5 & 100 & $3.4 \pm 0.27$ stuv & $6.4 \pm 0.163$ stuvw & $3.41 \pm 0.34$ opqrst \\
\hline 28. & $2.0+0.3$ & 15.7 & 100 & $3.6 \pm 0.27 \mathrm{rstu}$ & $6.8 \pm 0.249$ qrstu & $5.73 \pm 0.48 \mathbf{i j k l}$ \\
\hline 29. & $2.5+0.3$ & 14.1 & 100 & $4.6 \pm 0.16$ mnopq & $6.4 \pm 0.267$ stuvw & $5.89 \pm 0.64$ hijk \\
\hline 30. & $3.0+0.3$ & 14.3 & 100 & $5.2 \pm 0.25$ Imno & $8.4 \pm 0.221$ lmno & $6.85 \pm 0.44$ fghi \\
\hline 31. & $3.5+0.3$ & 13.5 & 100 & $5.3 \pm 0.34 \mathrm{lmn}$ & $9.6 \pm 0.371$ ghijk & $7.05 \pm 0.26$ efgh \\
\hline 32. & $4.0+0.3$ & 12.5 & 100 & $6.2 \pm 0.33 \mathbf{i j k}$ & $10.6 \pm 0.306 \mathbf{f g}$ & $7.74 \pm 0.36$ cdef \\
\hline 33. & $4.5+0.3$ & 11.6 & 100 & $6.4 \pm 0.43 \mathbf{h i j}$ & $10.8 \pm 0.416 \mathbf{f}$ & $5.64 \pm 0.48$ ijkk \\
\hline 34. & $5.0+0.3$ & 11.7 & 100 & $7.4 \pm 0.37$ efg & $12.2 \pm 0.467 \mathbf{e}$ & $3.0 \pm 0.38$ qrstuvw \\
\hline 35. & $5.5+0.3$ & 12.7 & 100 & $8.4 \pm 0.27 \mathbf{c d}$ & $12.3 \pm 0.359$ de & $2.37 \pm 0.29$ tuvwx \\
\hline 36. & $6.0+0.3$ & 11.9 & 100 & $9.8 \pm 0.29 \mathbf{a}$ & $12.1 \pm 0.476 \mathbf{e}$ & $1.53 \pm 0.14 \mathbf{x}$ \\
\hline 37. & $0.5+0.3$ & 18.1 & 100 & $2.2 \pm 0.25 \mathbf{x}$ & $5.1 \pm 0.233$ yzaa & $2.99 \pm 0.36$ qrstuvw \\
\hline 38. & $1.0+0.4$ & 17.2 & 100 & $2.4 \pm 0.16 \mathbf{w x}$ & $5.2 \pm 0.249$ xyzaa & $3.75 \pm 0.37$ nopqrs \\
\hline 39. & $1.5+0.4$ & 16.1 & 100 & $3.4 \pm 0.27$ stuv & $5.2 \pm 0.2$ xyzaa & $4.68 \pm 0.19$ klmno \\
\hline 40. & $2.0+0.4$ & 15.2 & 100 & $3.6 \pm 0.27$ rstu & $5.6 \pm 0.306 \mathbf{v w x y}$ & $5.7 \pm 0.31 \mathbf{i j k} \mathbf{l}$ \\
\hline 41. & $2.5+0.4$ & 14.9 & 100 & $4.6 \pm 0.16$ mnopq & $6.8 \pm 0.249$ qrstu & $7.76 \pm 0.48$ cdef \\
\hline 42. & $3.0+0.4$ & 14.5 & 100 & $5.2 \pm 0.25$ Imno & $8.2 \pm 0.359$ mnop & $7.57 \pm 0.41$ def \\
\hline 43. & $3.5+0.4$ & 12.7 & 100 & $5.2 \pm 0.25$ Imno & $9.2 \pm 0.249$ ijklm & $7.81 \pm 0.53$ cdef \\
\hline 44. & $4.0+0.4$ & 13.1 & 100 & $6.8 \pm 0.33$ fghi & $9.8 \pm 0.389$ fghij & $8.04 \pm 0.61$ cdef \\
\hline 45. & $4.5+0.4$ & 11.5 & 100 & $6.4 \pm 0.27 \mathbf{h i j}$ & $10.1 \pm 0.482$ fghi & $4.27 \pm 0.34$ mnopq \\
\hline 46. & $5.0+0.4$ & 11.2 & 100 & $7.4 \pm 0.34$ efg & $10.8 \pm 0.442 \mathbf{f}$ & $2.96 \pm 0.33$ qrstuvw \\
\hline 47. & $5.5+0.4$ & 12.9 & 100 & $8.4 \pm 0.45 \mathrm{~cd}$ & $10.8 \pm 0.291 \mathbf{f}$ & $2.21 \pm 0.30$ tuvwx \\
\hline 48. & $6.0+0.4$ & 13.7 & 100 & $8.5 \pm 0.43 \mathbf{a b}$ & $11.6 \pm 0.163 \mathbf{b c}$ & $2.04 \pm 0.27 \mathbf{u v w \mathbf { x }}$ \\
\hline 49. & $0.5+0.5$ & 17.9 & 100 & $2.4 \pm 0.27 \mathbf{w x}$ & $5.2 \pm 0.249$ xyzaa & $2.26 \pm 0.22$ tuvwx \\
\hline 50. & $1.0+0.5$ & 17.6 & 100 & $2.4 \pm 0.16 \mathbf{w x}$ & $5.6 \pm 0.163 \mathbf{v w x y}$ & $3.74 \pm 0.37$ nopqrs \\
\hline 51. & $1.5+0.5$ & 16.7 & 100 & $3.6 \pm 0.27 \mathrm{rstu}$ & $6.6 \pm 0.163$ rstuv & $4.47 \pm 0.39$ lmnop \\
\hline 52. & $2.0+0.5$ & 15.5 & 100 & $4.2 \pm 0.25$ pqrs & $6.2 \pm 0.2$ tuvwx & $5.27 \pm 0.35 \mathbf{j k l m}$ \\
\hline 53. & $2.5+0.5$ & 14.2 & 100 & $5.2 \pm 0.25$ lmno & $7.1 \pm 0.277$ qrst & $6.05 \pm 0.5$ ghij \\
\hline 54. & $3.0+0.5$ & 13.7 & 100 & $5.4 \pm 0.16 \mathrm{klm}$ & $7.4 \pm 0.306$ pqrs & $8.24 \pm 0.50$ cde \\
\hline 55. & $3.5+0.5$ & 13.6 & 100 & $6.4 \pm 0.27$ hij & $8.4 \pm 0.371$ lmno & $7.87 \pm 0.35$ cdef \\
\hline
\end{tabular}




\begin{tabular}{|c|c|c|c|c|c|c|}
\hline 56. & $4.0+0.5$ & 11.7 & 100 & $7.6 \pm 0.16$ def & $9.4 \pm 0.371$ hijkl & $7.94 \pm 0.38$ cdef \\
\hline 57. & $4.5+0.5$ & 12.5 & 100 & $7.6 \pm 0.27$ def & $9.8 \pm 0.327$ fghij & $9.77 \pm 0.63 \mathbf{a b}$ \\
\hline 58. & $5.0+0.5$ & 11.2 & 100 & $7.4 \pm 0.27$ efg & $10.6 \pm 0.34 \mathbf{f g}$ & $4.81 \pm 0.35$ jklmn \\
\hline 59. & $5.5+0.5$ & 12.7 & 100 & $8.1 \pm 0.28$ cde & $12.2 \pm 0.163$ cd & $2.28 \pm 0.23$ tuvwx \\
\hline 60. & $6.0+0.5$ & 13.5 & 100 & $9.6 \pm 0.37 \mathbf{a b}$ & $12.0 \pm 0.221 \mathbf{a}$ & $1.89 \pm 0.19 \mathbf{v w x}$ \\
\hline 61. & $0.5+0.6$ & 18.7 & 100 & $2.8 \pm 0.25$ uvwx & $4.8 \pm 0.133$ yzaа & $3.09 \pm 0.32$ qrstuv \\
\hline 62. & $1.0+0.6$ & 17.3 & 100 & $3.2 \pm 0.13$ tuvw & $5.4 \pm 0.163 \mathbf{w x y z}$ & $4.03 \pm 0.36$ mnopqr \\
\hline 63. & $1.5+0.6$ & 16.4 & 100 & $4.2 \pm 0.25$ pqrs & $5.8 \pm 0.327$ uvwxy & $4.99 \pm 0.38$ jkkmn \\
\hline 64. & $2.0+0.6$ & 15.6 & 100 & $3.8 \pm 0.25$ qrst & $5.1 \pm 0.233$ yzaa & $7.16 \pm 0.31$ efg \\
\hline 65. & $2.5+0.6$ & 14.7 & 100 & $5.1 \pm 0.31$ lmnop & $6.2 \pm 0.2$ tuvwx & $7.6 \pm 0.42$ def \\
\hline 66. & $3.0+0.6$ & 14.4 & 100 & $4.8 \pm 0.29$ mnop & $6.8 \pm 0.249$ qrstu & $8.74 \pm 0.54$ bcd \\
\hline 67. & $3.5+0.6$ & 13.9 & 100 & $5.8 \pm 0.29$ jkkl & $8.2 \pm 0.359$ mnop & $8.27 \pm 0.59$ cde \\
\hline 68. & $4.0+0.6$ & 13.4 & 100 & $6.0 \pm 0.26 \mathbf{i j k l}$ & $8.4 \pm 0.306$ lmno & $8.91 \pm 0.64$ bc \\
\hline 69. & $4.5+0.6$ & 12.2 & 100 & $9.8 \pm 0.42 \mathbf{a}$ & $12.6 \pm 0.427 \mathbf{a}$ & $9.02 \pm 0.65$ bc \\
\hline 70. & $5.0+0.6$ & 14.2 & 100 & $6.6 \pm 0.4$ ghij & $9.6 \pm 0.371$ ghijk & $6.76 \pm 0.37$ fghi \\
\hline 71. & $5.5+0.6$ & 13.7 & 100 & $8.4 \pm 0.43$ cd & $10.4 \pm 0.267$ fgh & $2.64 \pm 0.29$ stuvwx \\
\hline 72. & $6.0+0.6$ & 13.6 & 100 & $8.8 \pm 0.33$ bc & $10.8 \pm 0.327 \mathbf{b}$ & $1.9 \pm 0.23 \mathbf{v w x}$ \\
\hline
\end{tabular}

Values followed by same letters in each column are not significantly different $(\mathrm{p}<0.05)$ using DMRT 
Table.3 Effect of various concentrations of Auxins (IBA and NAA) added singly and in combinations in the MS medium on rooting in auxiliary explants of Aloe vera

\begin{tabular}{|c|c|c|c|c|c|}
\hline $\begin{array}{l}\text { Auxins } \\
\text { (mg/l) }\end{array}$ & $\begin{array}{c}\text { Days taken } \\
\text { in root } \\
\text { initiation }\end{array}$ & $\begin{array}{c}\text { Rooting } \\
\text { response } \\
(\%)\end{array}$ & $\begin{array}{l}\text { Number of } \\
\text { roots/explant }\end{array}$ & $\begin{array}{l}\text { Root length } \\
\text { (cm) }\end{array}$ & $\begin{array}{c}\text { Number of } \\
\text { shoot } \\
\text { buds/explant }\end{array}$ \\
\hline IBA 0.5 & 25.2 & 100 & $4.7 \pm 0.153$ bc & $2.74 \pm 0.142$ ef & $1.2 \pm 0.13 \mathbf{c}$ \\
\hline IBA 1.0 & 24 & 100 & $5.4 \pm 0.163 \mathbf{b}$ & $3.92 \pm 0.213 \mathrm{~cd}$ & $1.2 \pm 0.13 \mathbf{c}$ \\
\hline IBA 1.5 & 21.2 & 100 & $6.6 \pm 0.400 \mathbf{a}$ & $7.64 \pm 0.330 \mathbf{a}$ & $1.1 \pm 0.1 \quad$ c \\
\hline IBA 2.0 & 22.4 & 100 & $2.2 \pm 0.133 \mathbf{e}$ & $1.81 \pm 0.142 \mathbf{g}$ & $1.3 \pm 0.15 \mathbf{c}$ \\
\hline NAA 0.5 & 26.2 & 100 & $4.6 \pm 0.306$ bc & $5.64 \pm 0.279$ b & $2.1 \pm 0.28 \mathbf{b}$ \\
\hline NAA 1.0 & 25.4 & 100 & $4.1 \pm 0.539$ cd & $4.55 \pm 0.318$ c & $2.4 \pm 0.22 \mathbf{a b}$ \\
\hline NAA 1.5 & 20 & 100 & $3.6 \pm 0.340 \mathrm{~d}$ & $3.24 \pm 0.207$ de & $2.4 \pm 0.22 \mathbf{a b}$ \\
\hline NAA 2.0 & 20 & 100 & $3.4 \pm 0.221 \mathrm{~d}$ & $2.49 \pm 0.291 \mathbf{f g}$ & $2.7 \pm 0.15 \mathbf{a}$ \\
\hline \multicolumn{6}{|l|}{$($ IBA + NAA $)$} \\
\hline $0.5+0.5$ & 22.4 & 100 & $4.2 \pm 0.291$ bcd & $3.1 \pm 0.317 \mathbf{d}$ & $1.3 \pm 0.15 \mathbf{d}$ \\
\hline $0.5+1.0$ & 16.8 & 100 & $4.5 \pm 0.522$ abc & $4.2 \pm 0.384$ cd & $1.8 \pm 0.2 \quad$ cd \\
\hline $0.5+1.5$ & 14.2 & 100 & $4.8 \pm 0.467 \quad \mathbf{a b}$ & $5.6 \pm 0.45 \quad$ b & $2.1 \pm 0.23$ bc \\
\hline $0.5+2.0$ & 13.6 & 100 & $5.8 \pm 0.742 \quad \mathbf{a}$ & $8.3 \pm 0.448 \mathbf{a}$ & $2.3 \pm 0.26 \mathbf{a b}$ \\
\hline $2.0+0.5$ & 15.6 & 100 & $2.8 \pm 0.327 \quad$ d & $6.2 \pm 0.429 \mathbf{b}$ & $2.2 \pm 0.25 \mathbf{a b}$ \\
\hline $2.0+1.0$ & 12.8 & 100 & $3.9 \pm 0.458$ bcd & $5.8 \pm 0.404 \mathbf{b}$ & $1.8 \pm 0.2 \quad$ cd \\
\hline $2.0+1.5$ & 10.9 & 100 & $3.4 \pm 0.340$ bcd & $5.5 \pm 0.390 \quad \mathbf{b}$ & $2.5 \pm 0.22 \mathbf{a b}$ \\
\hline $2.0+2.0$ & 10.6 & 100 & $3.1 \pm 0.379$ cd & $5.0 \pm 0.377 \quad$ bc & $2.8 \pm 0.13$ a \\
\hline
\end{tabular}

Values followed by same letters in each column are not significantly different $(\mathrm{p}<0.05)$ using DMRT

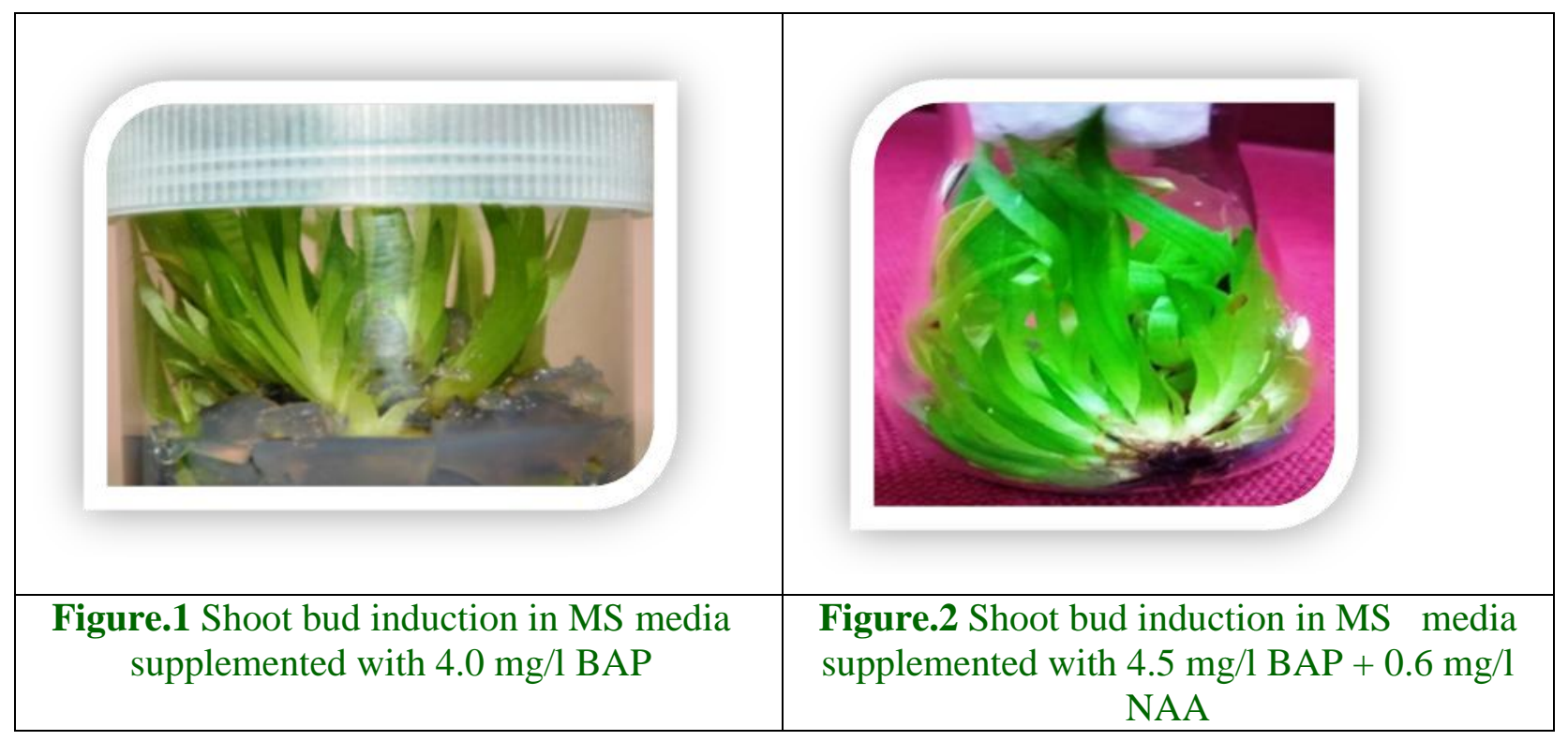




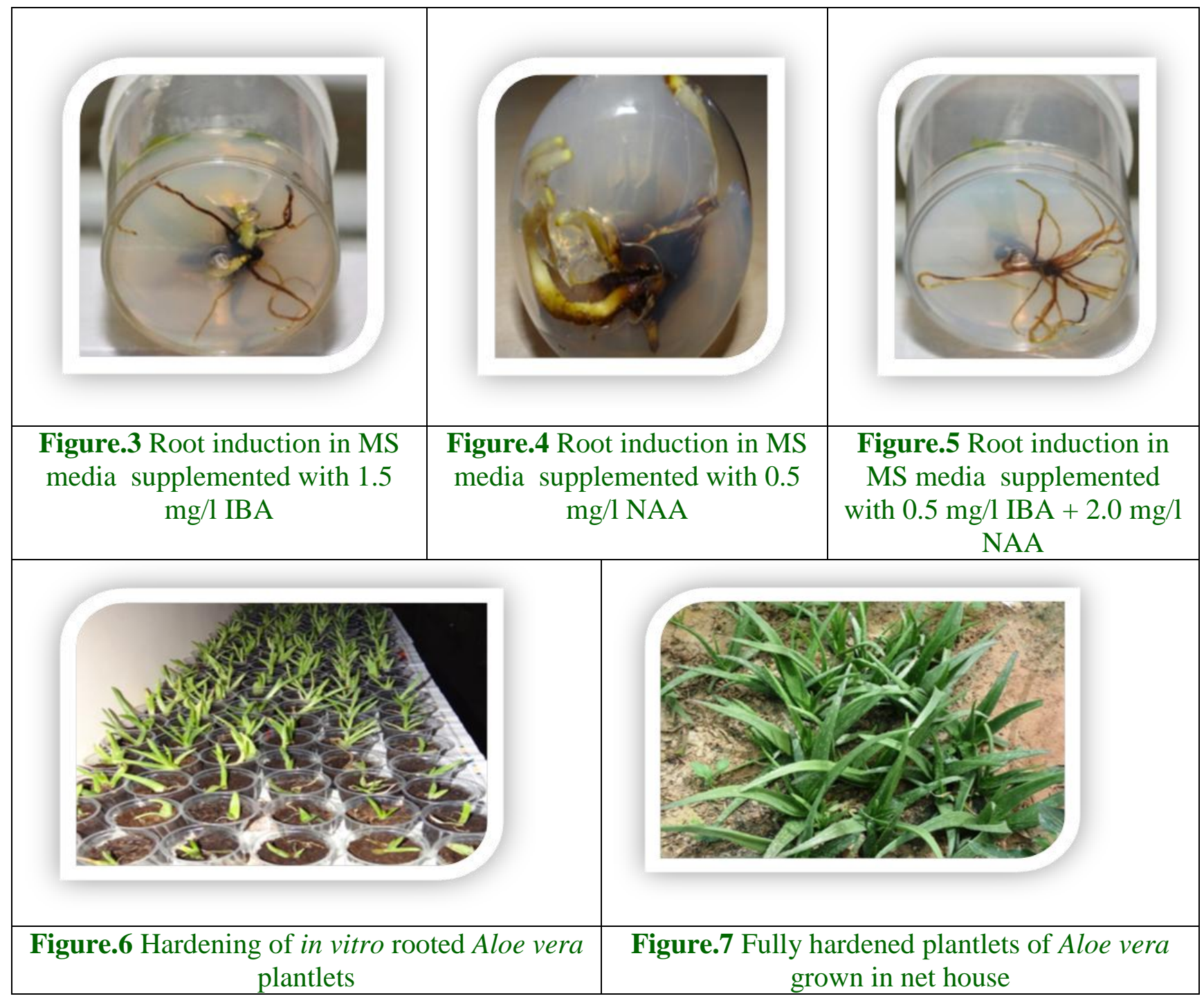

In the present investigation we did not investigate all the factors which in one way or other way block or stimulate micropropagation potential in Aloe vera, thus in the light of these factors we feel the prolonged and intensive in vitro efforts are needed to exploit the micropropagation in Aloe vera.

However, results of the present study may be recommended to develop protocols for mass multiplication of Aloe vera to meet the demand of farmers and pharmaceutical industries.

\section{Acknowledgements}

The author acknowledges the support from all the staff of Tissue culture laboratory and department of Plant Breeding and Genetics, S. K. N. Agriculture University, Jobner, Rajasthan (India) for conducting the experiment.

\section{References}

Abrie, A. and Staden, J.V. 2001. Micro propagation of endangered Aloe polyphylla. Plant Growth Regulation. 33: 19-23.

Ahmed, S., Kabir, A. H., Ahmed, M. B., 
Razy, M. A. and Ganesan, S. 2007. Development of rapid micropropagation method of Aloe vera (L).Sjemenarstvo, 24: 2

Bhandari, Aravind Kumar, Negi JS, Bisht VK and Bharti MK. 2010. In vitro propagation of Aloe vera - A plant with Medicinal properties. Nature and Science. 8: 8 .

Dwivedi, N. K., Indiradevi, A., Asha, K. I., Nair, R. A. and Suma, A. 2014. A protocol for micropropagation of Aloe vera L. (Indian Aloe)- A miracle plant, Research in Biotechnology,5(1): 1-5.

Eufrocinio, C. M. and Malasa, A. B. 2005.Tissue culture for the rapid clonal propagation of Aloe barbadensis Miller.The Philippines Agricultural Scientist, 88(1): 167-170.

Gomez, K. A., Gomez, A. A. 1984. Statistical Procedures for Agricultural Research. IRRI, Phillipines, 2nd edition.

Gupta, S., Sahu, P.K., Sen, D.L. and Pandey, P. 2014. In-vitro Propagation of Aloe vera (L.) Burm. $f$ British Biotechnology Journal 4(7): 806-816

Hashemabadi, D. and Kaviani, B. 2008.Rapid micro-propragation of Aloe vera $\mathrm{L}$. via shoot multiplication. African Journal Biotechnology, 7(12): 1899-1902.

Kalimuthu, K., Vijayakumar, S., Senthilkumar, R.R. and Suresh K. M. 2010. Micropropagation of Aloe vera Linn. - A medicinal plant. International Journal of Biotechnology and Biochemistry, 6(3): 405-410.

Kawai, K., Beppu, H., Koike, T., Fujita, K. and Marunouchi, T. 2006. Tissue culture of Aloe arborescens Miller var. natalensis Berger.Phytotherapy Research, 7(7): S5-S10.

Keijzer, C.J. and Cresti, M. 1987. A comparison of anther tissue development in male sterile Aloe vera and male fertile Aloe ciliaris. Annals of Botany, 59 : 533- 542.
Khanam, M. and Sharma, G. K. 2014.Rapid in vitro propagation of Aloe vera $\mathrm{L}$. with some growth regulators using lateral shoots as explants. World Journal of Pharmacy and Pharmaceutical Sciences, 3(3): 2278-4357.

Kiran, S., Tirkey, A., Jha, Z. and Porte, S. S. 2017. In vitro regeneration of Aloe vera (Aloe barbadensis Mill).International Journal of Current Microbiology and Applied Sciences, 6(11): 1829-1834.

Meyer HT and Staden JV. 1991.Rapid in vitro propagation of Aloe barbadensis Mill. Plant Cell Tissue Organ Cult. 26: 171176.

Murashige T, Skoog F. 1962. A revised medium for rapid growth and bioassays with tobacco tissue cultures. Physiol. Plant.;115:493-497.

Nayanakantha, N. M. C., Singh, B. R. and Gupta, A. K. 2010. Assessment of genetic diversity in Aloe germplasm accessions from India using RAPD and morphological markers. Biological Science,39(1): 1- 9.

Rathore, M. S., Chikara, J. and Shekhawat, N. S. 2011. Plantlets regeneration from callus cultures of selected genotype of Aloe vera L.- An ancient plant for modern herbal industries. Applied Biochemistry and Biotechnology,165 (7): 860-868.

Snedecor GW and Cochran WG. 1972. Statistical method $6^{\text {th }}$ edition, Lowa State University Press, Lowa, 258-298.

Souza, V. and Lorenzi, H. 2005. Botânica Sistemática: Guia ilustrado para identificacxão das famı'lias de Angiospermas da flora brasileira, baseado em APG II. Instituto Plantarum, Nova Odessa, 640.

Yadav, L. 2008. Standardization of micropropagation protocol in Ghritkumari (Aloe barbadensis Mill.). M.Sc. Thesis, Rajasthan Agricultural University, Bikaner. 


\section{How to cite this article:}

Mohan Lal Jakhar, Divya Dixit, Sarfraz Ahmad, Mahaveer Prasad Ola, Manohar Ram and Hari Ram Jat. 2020. Influence of Plant Growth Regulators on Micropropagation of Gwarpatha [Aloe vera (L.) Burm.]. Int.J.Curr.Microbiol.App.Sci. 9(03): 1792-1803.

doi: https://doi.org/10.20546/ijcmas.2020.903.208 